\title{
CRITÉRIOS DE QUALIDADE EM PERIÓDICOS CIENTÍFICOS
}

\author{
Paula Caroline Schifino Jardim Passos* \\ Jaire Ederson Passos ${ }^{* *}$ \\ Sônia Elisa Caregnato \\ Tânia Luisa Koltermann da Silva ${ }^{* *+*}$
}

RESUMO

\begin{abstract}
A avaliação de periódicos científicos tem sido preocupação dos pesquisadores desde meados do século $X X$. Os critérios de avaliação inicialmente propostos consideravam a qualidade das revistas científicas em seu suporte original, o impresso. Porém, com o tempo, essas revistas adotaram preponderantemente $\circ$ meio digital para publicação. Neste contexto, interessa à presente pesquisa investigar em que medida a avaliação dos periódicos científicos tem acompanhado o avanço tecnológico, abrangendo também aspectos relacionados ao suporte eletrônico. A questão que norteia a pesquisa é a seguinte: como as metodologias de avaliação de revistas científicas comtemplam critérios referentes ao meio digital? Para responder a esta pergunta, identificouse, nas publicações relativas ao tema, critérios de qualidade de revistas científicas associados às especificidades da mídia eletrônica. $O$ levantamento de dados foi efetuado nas bases Web of Science, Science Direct e Brapci, na Biblioteca Digital Brasileira de Teses e Dissertações, no Banco de Tese e no Google Acadêmico. Como recurso para visualização dos resultados, apresenta-se um quadro comparativo das metodologias de avaliação que destaca a presença dos critérios: meio impresso, meio eletrônico, hipertexto, hipermídia, usabilidade, acesso aberto, arquitetura da informação e recursos para interação e colaboração em rede. $O$ quadro também informa sobre os autores que deram origem aos estudos relacionados e classifica as metodologias como qualitativa ou quantitativa. A pesquisa demonstra uma tendência de adequação das metodologias de avaliação de revistas científicas ao contexto digital e apresenta as áreas que ainda requerem estudos aprofundados.
\end{abstract}

\footnotetext{
Palavras-chave: Periódico científico. Avaliação de qualidade. Arquitetura da informação.
}

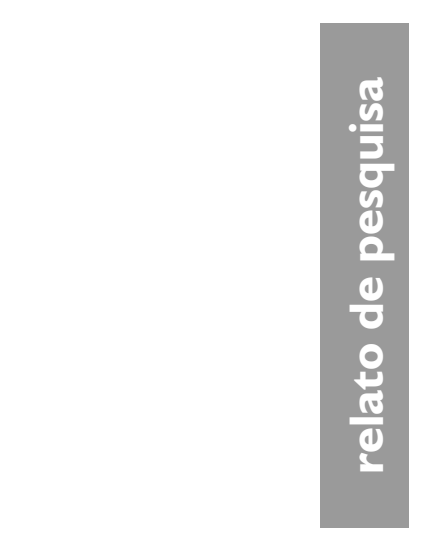

* Doutora em Comunicação e Informação. Pesquisadora de Pós-doutorado no Programa de Pós-Graduação em Comunicação e Informação da Universidade Federal do Rio Grande do Sul, Brasil. E-mail: paulacarolinejardim@gmail.com.

** Professor assistente do Departamento de Design e Expressão Gráfica da Faculdade de Arquitetura da Universidade Federal do Rio Grande do Sul, Brasil. Doutorando no Programa de Pós-Graduação em Design da Universidade Federal do Rio Grande do Sul, Brasil. E-mail: jairepassos@gmail.com.

*** Doutora em Information Studies pela University of Sheffield, Inglaterra. Professora Associada do Departamento de Ciências da Informação da Universidade Federal do Rio Grande do Sul, Brasil. E-mail: sonia.caregnato@ufrgs.br.

***** Professora Associada do Departamento de Design e Expressão Gráfica da Faculdade de Arquitetura da Universidade Federal do Rio Grande do Sul (UFRGS). Docente Permanente do Programa de Pós-Graduação em Design da Universidade Federal do Rio Grande do Sul, Brasil.

E-mail: tania.koltermann@ufrgs.br.

\section{INTRODUÇÃO}

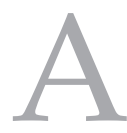

publicação, em 1934, de Bradford's Law of Mathematics, confere a Samuel Clement Bradford o título de precursor dos estudos de avaliação de periódicos científicos. Nas décadas subsequentes, Alan Pritchard dá nome de "bibliometria" às pesquisas estatísticas em bibliografia (GUÉDON, 2000). Já na década de 1960, Eugene Garfield, fundador e presidente do Institute for Scientific Information (ISI), realiza análises estatísticas em larga escala de citações de periódicos e chega à conclusão de que a maioria das citações são atribuídas a relativamente poucas revistas, enquanto uma minoria de 
citações é distribuída por muitas revistas. Esse trabalho é considerado como a origem das avaliações de revistas científicas (YU et al., 2009).

Ainda na década de 1960, diversos programas de avaliação de revistas passaram a ser desenvolvidos por instituições como a Organização das Nações Unidas para Educação, Ciência e Cultura (UNESCO), o Centro LatinoAmericano e do Caribe de Informação e Ciências da Saúde (BIREME), o Instituto Brasileiro de Informação em Ciência e Tecnologia (IBICT), a Fundação de Apoio à Pesquisa do Estado de São Paulo (FAPESP), o Conselho Nacional de Desenvolvimento Científico e Tecnológico (CNPq), a Financiadora de Estudos e Projetos (FINEP) e a Coordenação de Aperfeiçoamento de Pessoal de Nível Superior (CAPES), entre outros (STUMPF, 2003). Essas iniciativas buscaram estabelecer critérios de qualidade para diversos propósitos, como a distinção dos principais periódicos de cada área, admissão em bases dados e classificação para o recebimento de apoio financeiro.

Os primeiros métodos de avaliação eram compostos por critérios de qualidade descritos com vistas às revistas impressas. A partir do século XXI, algumas pesquisas passaram a atualizar os modelos de avaliação considerando também aspectos relacionados aos periódicos científicos eletrônicos. O presente artigo resgata esses estudos com intuito de investigar quais aspectos relacionados ao contexto digital têm sido contemplados. Para tanto, o relato que segue busca identificar, nas publicações disponíveis, critérios de qualidade associados às especificidades da mídia eletrônica, como arquitetura da informação, design de interface, apresentação visual no contexto digital, usabilidade, acesso aberto, hipermídia e recursos para interação e colaboração em rede.

\section{METODOLOGIA}

Este estudo consiste em uma pesquisa bibliográfica em trabalhos científicos sobre o tema avaliação de revistas acadêmicas. O levantamento de dados foi efetuado nas bases de dados Web of Science, Science Direct e Brapci, na Biblioteca Digital Brasileira de
Teses e Dissertações, no Banco de Teses da CAPES e no Google Acadêmico. Os dados apresentados foram extraídos das publicações que mais se aproximam do tema desta pesquisa e que apresentaram algum grau de novidade em relação às publicações anteriores. A questão que norteia a pesquisa é a seguinte: como as metodologias de avaliação de revistas científicas tem contemplado critérios referentes ao meio digital?

A análise realizada a partir das metodologias de avaliação destaca critérios voltados às especificidades dos periódicos científicos eletrônicos. Como recurso para visualização dos resultados, esta pesquisa apresenta um quadro comparativo das metodologias de avaliação, destacando os que critérios citados em cada uma delas, conforme os itens relacionados: meio impresso, meio eletrônico, hipertexto, hipermídia, usabilidade, acesso aberto, arquitetura da informação e recursos para interação e colaboração em rede. $\mathrm{O}$ quadro também informa origem dos autores e a classificação das metodologias como qualitativa ou quantitativa.

\section{INICIATIVAS PARA AVALIAÇÃO DE PERIÓDICOS CIENTÍFICOS}

Em 1964, um grupo de pesquisadores, patrocinados pela UNESCO, reuniu-se em Porto Rico para estudar os problemas dos periódicos científicos e técnicos latino-americanos. Nessa reunião, foram estabelecidos critérios para classificação dos periódicos, conferindo-lhes qualificações entre deficiente a excelente. Esse modelo de avaliação desenvolvido para a UNESCO foi adaptado no Brasil por um grupo de pesquisadores do IBICT. Nele, são estabelecidos critérios de avaliação quanto a normalização, tempo de existência da revista, periodicidade, indexação, difusão, colaboração em termos de autoria e autoridade da comissão editorial. Cada um desses critérios possuía variáveis e pontuações correspondentes, por exemplo: em periodicidade, a revista que fosse publicada semestralmente receberia 1 ponto, enquanto a revista trimestral receberia 3 pontos. Dessa forma, eram atribuídas pontuações às revistas (BRAGA; OBERHOFER, 1982). A Figura 1 foi adaptada com base nesse modelo. 
Figura 1: Modelo para avaliação de periódicos científicos e técnicos

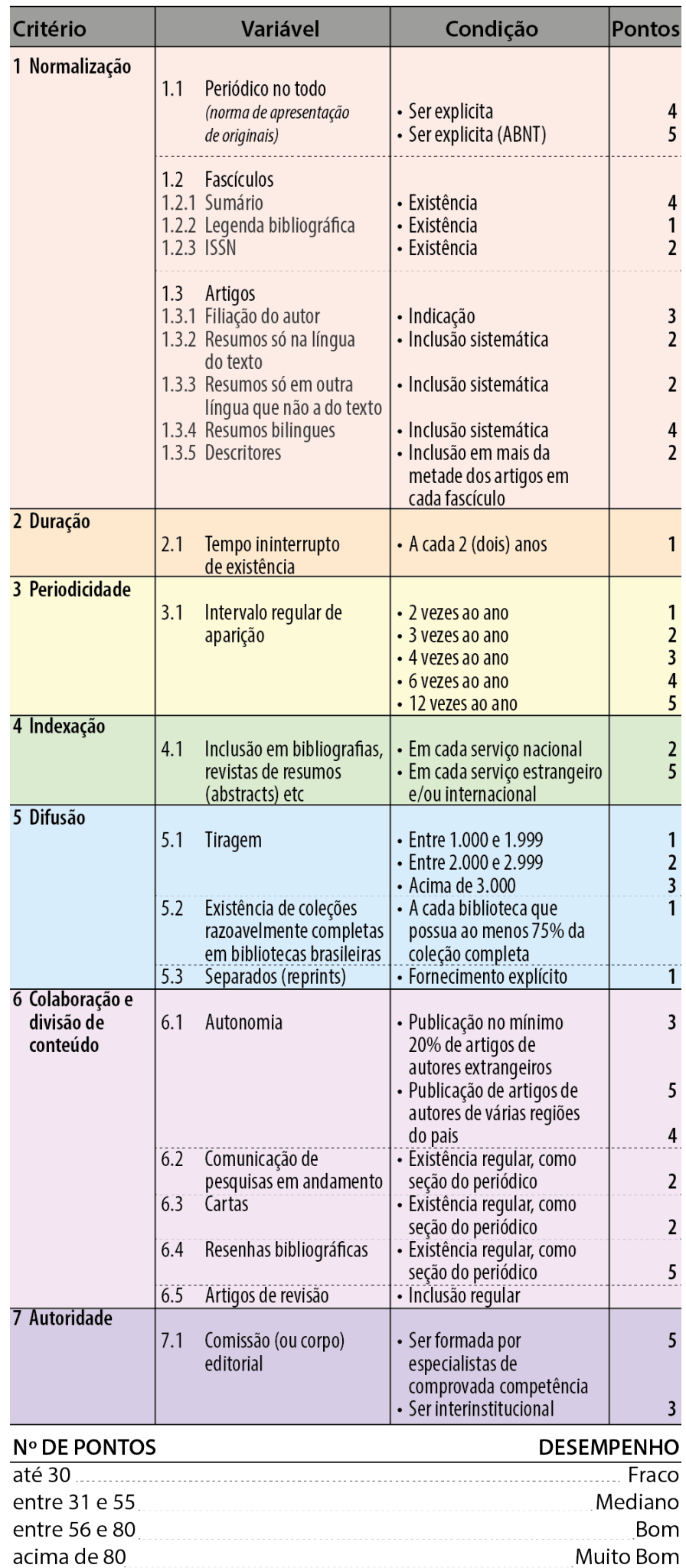

Fonte: Adaptado de Braga e Oberhofer (1982) 
Desde então, o modelo de Braga e Oberhofer (1982) tem servido de fundamento para diversas pesquisas interessadas na avaliação de periódicos. Yahn (1985) o adapta para proceder avaliações de revistas na área da agricultura e agrega a este, a pesquisa qualitativa quanto às impressões dos pesquisadores da área. Castro, Ferreira e Vidili (1996) também adaptam o modelo, dessa vez para avaliação de periódicos latino-americanos na área da ciência da saúde indexados nas bases Medlars Online (Medline) e Literatura Latino-Americana em Ciências da Saúde (LILACS), criada pelo BIREME. Esse projeto teve apoio do Programa de Desenvolvimento Científico da Organização Pan-Americana da Saúde (OPAS) e objetivou a definição de um formulário para análise dos periódicos que se candidatavam à inclusão na base LILACS.

Com intuito de avaliar e classificar os periódicos brasileiros, com vistas à priorização de apoio financeiro da FAPESP, Krzyzanowski, Krieger e Duarte (1991) desenvolvem um método de avaliação de mérito (referente ao conteúdo publicado). Nesse processo, especialistas de diversas áreas deveriam atribuir conceitos aos periódicos com base em um questionário predefinido pelos autores. Essa pesquisa deu origem ao Núcleo Básico de Periódicos Científicos Brasileiros Correntes. Posteriormente, Krzyzanowski e Ferreira (1998) atualizam essa listagem avaliando os periódicos financiados pelo FINEP, pelo CNPq e pela FAPESP. A nova proposta aplicava o método de Krzyzanowski, Krieger e Duarte (1991), para avaliação de mérito, aliado ao modelo de Braga e Oberhofer (1982) para avaliação de desempenho (ou da forma) das revistas.

Esses modelos de avaliação iniciais foram de várias formas adaptados, como no caso da pesquisa de Yamamoto et al. (2002), que relatam a avaliação de revistas científicas brasileiras na área da Psicologia. Trata-se de um estudo realizado pela Associação Nacional de Pesquisa e Pós-Graduação em Psicologia (Anpepp), sob encomenda da Capes, que teve uma primeira versão publicada três anos antes (YAMAMOTO; SOUZA; YAMAMOTO, 1999). A inovação principal dessa pesquisa foi a inserção de uma lista de orientações para o preenchimento da ficha de avaliação.

Como parte de um esforço para aperfeiçoar o sistema de avaliação da pósgraduação brasileira, a Capes, em 1998, propõe o desenvolvimento da base Qualis. Em relação às revistas científicas, o objetivo da Capes foi a "[...] construção de indicadores de produção científica fundamentados na qualidade das revistas científicas utilizadas pelos programas de pós-graduação, devendo estas receber uma classificação conforme critérios definidos pelas comissões de áreas". Inicialmente, foram reunidos todos os veículos utilizados pelos programas de pós-graduação nacionais a partir dos relatórios que estes enviam à Capes. A seguir, essas publicações foram avaliadas conforme critérios definidos para cada área do conhecimento (SOUZA; PAULA, 2002, p.8).

Atualmente, o Qualis-Periódicos é definido como "[...] o conjunto de procedimentos utilizados pela Capes para estratificação da qualidade da produção intelectual dos programas de pósgraduação". Anualmente, a Capes divulga uma lista com a classificação dos periódicos em cada área, enquadrando-os em estratos indicativos da qualidade - A1, o mais elevado; A2; B1; B2; B3; B4; B5; C - com peso zero. Um mesmo periódico pode ser classificado em mais de uma área, recebendo avaliações diferentes. Pode-se consultar a classificação de um periódico por meio do aplicativo WebQualis (CAPES, 2015).

Na mesma década, em 1996, uma parceria entre o BIREME e a FAPESP dá origem a Scientific Electronic Library Online (SciELO), uma biblioteca virtual que organiza e publica na internet textos completos de revistas científicas. A partir de 2002, o projeto passou a receber também o apoio do CNPq (SCIELO, 2015). Segundo a Revista Pesquisa Fapesp (2002), o intuito do projeto foi aumentar a visibilidade e a acessibilidade das revistas científicas brasileiras, uma vez que a maioria não estava indexada em bases de dados internacionais.

Já o interesse central da BIREME, segundo Packer (1998), foi o desenvolvimento de uma metodologia para publicação eletrônica, cuja aplicação pudesse complementar a metodologia de registro bibliográfico e a indexação utilizada na produção descentralizada da base de dados bibliográficos LILACS. Percebe-se aqui que iniciam as avaliações considerando também a publicação de revistas no contexto digital.

A inclusão dos periódicos na SciELO é feita por meio de revisão e de aprovação de consultores da área. Os critérios de qualidade considerados para inclusão das revistas na SciELO envolvem desde o número de citações 
de seus trabalhos, passando pela prontidão na publicação, até aspectos técnicos formais (GREENE, 2000). De caráter cooperativo, a SciELO possui metodologia para a preparação, armazenamento, disseminação e avaliação da produção científica em formato eletrônico.

Em 1998, os critérios de seleção para a base do ISI já consideravam também o suporte eletrônico. Para essa avaliação das revistas, muitos dos fatores considerados envolviam padrões básicos de apresentação, de conteúdo editorial, de internacionalidade dos autores e de citação de dados associada a esses autores. Conforme James Testa, gerente editorial do ISI, os fatores não eram considerados de forma isoladas, mas sempre combinados e interrelacionandos, o que promovia a avaliação da revista na sua totalidade (TESTA, 1998).

Outras pesquisas sobre avaliação de revistas, ainda do final do século XX, que cabe citar aqui, são de Martins (1986) e López-Cózar (1997), que tomam como critérios para avaliação as regras de normalização da International Organization for Standardization (ISO). Martins (1986) avalia o grau de normalização de 224 periódicos na área de ciência e de tecnologia a partir das normas da Associação Brasileira de Normas Técnicas (ABNT). Já López-Cózar (1997) avalia 205 revistas espanholas da área de ciência da saúde em relação às normas internacionais de apresentação.

\section{AVALIAÇÕES QUALITATIVAS QUANTITATIVAS}

O estudo de Chressanthis e Chressanthis (1993) mostra que, no século XX, a qualidade das revistas era medida principalmente de forma quantitativa, utilizando-se critérios como o número de artigos e de páginas por volume, a frequência de publicação, a circulação anual, e a idade das revistas. Stumpf (2003) explica que para realização de avaliações quantitativas são definidos parâmetros a partir dos aspectos intrínsecos e extrínsecos das revistas.

Em relação às características intrínsecas, as metodologias de avaliação podem indagar sobre o conteúdo dos artigos, sobre a reputação da instituição publicadora, sobre a composição do conselho editorial ou científico, sobre o sistema de seleção de originais, sobre os autores, sobre a difusão geral ou circulação da revista, sobre a indexação por serviços bibliográficos nacionais ou internacionais, sobre as medidas de citações e sobre o fator de impacto. Os critérios de qualidade extrínsecos envolveriam a periodicidade e a regularidade da publicação, o respeito às normas de apresentação e de padronização, o tempo de duração da publicação, a tiragem, a quantidade média de artigos que recebe e que publica em cada fascículo, bem como a correta apresentação gráfica, entre outros (STUMPF, 2003).

A pesquisa de Stumpf (2003) avalia vinte e seis revistas acadêmicas da área de Comunicação com base na opinião de docentes e pesquisadores ligados aos Programas de Pós-Graduação brasileiros. Esse foi um estudo exploratório com abordagem quantitativa. O instrumento utilizado para a coleta de dados foi um formulário onde os sujeitos atribuíram conceitos para as seguintes variáveis: avaliação geral da revista, contribuição para a área, prestígio junto à comunidade acadêmica, qualidade do conteúdo dos artigos, rigor na avaliação dos originais, regularidade da publicação, apresentação gráfica e distribuição. As revistas selecionadas para análise foram aquelas que eram conhecidas por, pelo menos, a metade dos respondentes. Como resultado, a pesquisa aponta as oito revistas mais conceituadas na área. $\mathrm{O}$ instrumento utilizado para pesquisa pode ser visto na Figura 2.

Figura 2: Modelo para avaliação de periódicos científicos

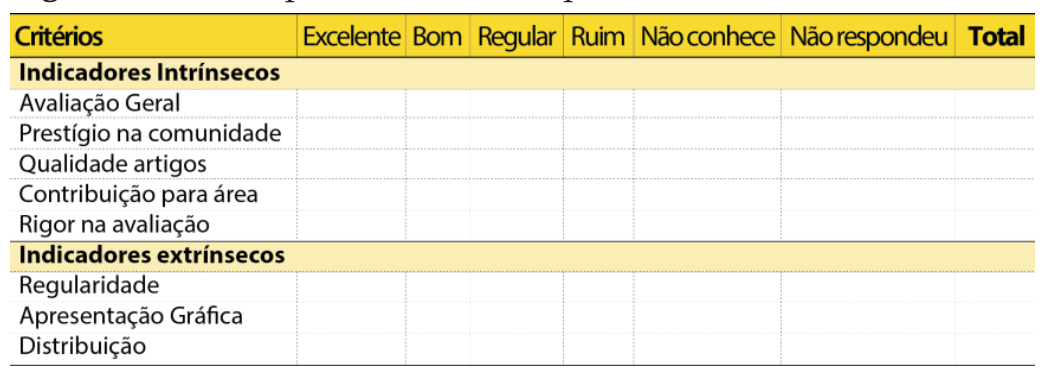

Fonte: Adaptado de Stumpf (2003) 
Stumpf (2003) observa que a literatura internacional, até aquele momento, voltava principal atenção ao processo de avaliação dos originais pelos pares, já que este tem sido considerado ponto fundamental para o controle de qualidade das revistas e para a manutenção do elevado padrão de qualidade da ciência. Atualmente, entretanto, o ranking das revistas parece ser a preocupação principal em termos internacionais. Muitas das pesquisas encontradas sobre o tema buscam estabelecer um ranking das revistas para informar aos pesquisadores quais veículos proporcionarão maior visibilidade ao trabalho, como exemplificam os trabalhos apresentados a seguir.

Nos Estados Unidos, Zsidisin et al. (2007) constroem uma ferramenta para avaliação de revistas na área de negócios (Purchasing and Supply Management, PSM). A ferramenta foi desenvolvida baseada em entrevistas com especialistas da área e aplicada aos pesquisadores usuários das revistas. A intenção foi avaliar as revistas quanto a sua qualidade, sua reputação e sua relevância para as áreas da prática profissional e da pesquisa. A pesquisa não considerou questões de estrutura ou interface.

Garand et al. (2009), por sua vez, avaliam as revistas em Ciência Política nos países Estados Unidos, Canadá e Inglaterra. Eles realizam entrevistas com os pares a fim de estabelecer um ranking de reputação das revistas da área. Os resultados da pesquisa mostram que os periódicos mais importantes em Ciência Política são aqueles que são altamente considerados pela qualidade do trabalho que eles publicam e, ao mesmo tempo, altamente visíveis para o grupo mais amplo de cientistas políticos.

Bolaños-Pizarro et al. (2009) procedem uma avaliação para obtenção de ranking de revistas na área de sistema cardiovascular inclusas na base de dados Índice Médico Espanhol (IME) 2006. Para tanto, constroem uma ferramenta que conta com 64 características que atendem a cinco grupos: critérios para a apresentação das revistas, critérios para a apresentação de artigos, comissões editoriais e cientistas, características do conteúdo, critérios de transmissão. Esse instrumento é composto a partir dos critérios de qualidade para a seleção de revistas na base de dados IME. Nos critérios de transmissão, a pesquisa considera se a revista conta com versão eletrônica; não considera, entretanto, arquitetura da informação ou questões de interface.
Semelhantemente, a pesquisa de Cheng et al. (2010) visa estabelecer uma classificação de revistas, desta vez na China. Para tanto, eles desenvolvem um algoritmo para cálculo de prestígio das publicações. Eles explicam que essa é uma alternativa ao cálculo de citações para o estabelecimento de fator de impacto. Embora importante, o cálculo de citações e o fator de impacto são considerados limitados, haja vista que valoram igualmente todos os artigos de uma mesma revista - independente de sua qualidade. Foram classificados todos os trabalhos da área de física, publicados entre 2004 e 2006 no Chinese Scientific and Technology Papers and Citation Database (CSTPCD). Eles concluem que o algoritmo desenvolvido se mostrou favorável para a definição de autoridades das revistas.

Como último exemplo de autores que aplicaram métodos quantitativos, pode-se citar Gemser e Friedman (2012), que realizam um levantamento com pesquisadores da área do design para elencar as principais revistas da área. Os critérios foram popularidade entre os pesquisadores da área e posição média no ranking de indexação. Sendo o design uma área recente, muitos pesquisadores optam por publicar em revistas de outras áreas, que já sejam reconhecidas. A ideia desses autores australianos foi indicar aos demais pesquisadores em design quais as revistas principais da área - mesmo que essas não contem com um fator de impacto elevado. Interessante observar que sua avaliação também não declara levar em consideração itens de apresentação visual aplicados ao contexto digital. Talvez o motivo seja que as revistas na área do design em geral já contam com elaboração visual apurada, então esse não seria um fator de distinção.

Alguns autores, por outro lado, têm se dedicado à avaliação de revistas por meio de pesquisas qualitativas. Já na década de 1990, Gorman (1999) colocava as medidas qualitativas como as mais adequadas para atribuição de excelência às revistas, considerando que os fatores avaliados eram qualitativos. Contemporâneos a esse autor, Day e Peter (1994) aplicaram métodos qualitativos na avaliação da qualidade de revistas na Inglaterra. Eles entrevistaram autores, editores, consultores editoriais e leitores de revistas científicas perguntando-lhes sobre os atributos de qualidade que mais valorizavam.

Para orientar sua pesquisa, Day e Peter (1994) compuseram uma lista de critérios 
separados pelos tópicos prestígio, conteúdo e apresentação: era considerado o prestígio de autores, de editores e do conselho editorial; sobre o conteúdo, avaliou-se as aplicações práticas da pesquisa, a originalidade, a clareza, a legibilidade, o rigor científico, a contribuição ao conhecimento, os recursos aplicados e as perspectivas de internacionalização; em termos de apresentação, foram considerados itens como projeto de capa, tipografia, leiaute, uso de gráficos e facilidade proporcionada pelo projeto na busca das referências.
A pesquisa de Day e Peter (1994), mesmo que dedicada principalmente a revistas impressas, demonstra um início de preocupação com itens que seriam desenvolvidos mais profundamente com o advento das pesquisas em arquitetura da informação. A preocupação com a capa pode ser equiparada à identificação da revista online. A busca das referências, bem como a disposição dos elementos visuais gráficos e tipográficos previstos no planejamento de arquitetura. A Figura 3 apresenta esse modelo de avaliação.

Figura 3: Modelo para avaliação de periódicos científicos

\begin{tabular}{|l|l|l|}
\hline \multicolumn{1}{|c|}{ A) Prestígio } & B) Conteúdo & \multicolumn{1}{|c|}{ C) Apresentação } \\
\hline A1 Prestígio dos autores & B1 Aplicações práticas & C1 Capa do projeto \\
A2 Prestígio do editor & B2 Originalidade & C2 Tipografia e leiaute \\
A3 Prestigio do conselho & B3 Clareza e legibilidade & C3 Facilidade de referência \\
consultivo editorial & B4 Rigor científico \\
& $\begin{array}{l}\text { B5 Contribuição ao } \\
\text { conhecimento } \\
\text { B6 Mix de recursos } \\
\text { B7 Perspectivas } \\
\text { internacionais }\end{array}$ & \\
& & \\
\hline
\end{tabular}

Fonte: Adaptado de Day e Peter (1994)

Pouco depois, na Nigéria, Nkereuwem (1997) utilizou método semelhante. Ele perguntou a bibliotecários de todo o país não só quais as revistas que eles consultavam, mas também qual sua impressão sobre a qualidade dessas revistas. O resultado do trabalho consiste em escores de impacto e em um ranking das principais revistas da área para sua região. Anderson (1997) e Gorman e Calvert (2003) também realizam pesquisas qualitativas sobre revistas por meio de consulta aos editores.

$\mathrm{Na}$ Índia, Mukherjee (2010) também defende a avaliação qualitativa de revistas científicas. Ele opta por partir dos critérios de admissão das principais bases internacionais para desenvolver sua ferramenta de análise de revistas. Seu estudo compara uma seleção de revistas de acesso aberto com outras de acesso mediante assinatura e as avalia quanto a disponibilidade, autoridade, política de revisão, âmbito de aplicação, qualidade dos artigos, formato de página, disponibilidade de hiperlinks, política de atualização, motor de busca e problemas diversos.

Os itens avaliados por Mukherjee (2010) que mais interessam à presente pesquisa são o formato de página (que considera se a revista usa principalmente texto, ou apresenta também gráficos), os formatos dos arquivos (gif, png, jpg) e a quantidade desses arquivos. Ele considera ainda os formatos de arquivos aplicados - se pdf ou html/xml. No critério hiperlink, verifica-se se os hiperlinks encaminham do texto para outros textos, para referências e notas, para tabelas e figuras e para apêndice. Também é verificado se os hiperlinks das referências encaminham para as notas. Ainda se observa se há links para outros jornais de acesso aberto. Além disso, o critério motor de busca investiga quais os tipos de busca disponíveis. Esse trabalho já traz questões das revistas eletrônicas, que serão abordadas no próximo item. 


\section{AS PESQUISAS COM FOCO NA AVALIAÇÃO DE REVISTAS CIENTÍFICAS ELETRONICAS E ONLINE}

O foco principal deste relato é identificar elementos que contribuam para avaliação de revistas científicas eletrônicas. Como foi comentado anteriormente, desde os primeiros processos de avaliação, é possível encontrar critérios que se refiram a questões estruturais, quanto questões de apresentação do conteúdo. A partir, no entanto, da transposição do suporte impresso para o eletrônico, é possível perceber um despertar dos autores para as questões de interface e arquitetura.

No ano de 2002, dois trabalhos apresentam inovações nos processos de avaliação provocadas pelo advento do periódico eletrônico online: Fachin (2002) e Sarmento e Souza (2002). Fachin (2002) elabora um modelo de avaliação da padronização de periódicos científicos online brasileiros. Esse modelo tem base em Yahn (1985), Ferreira (2001) e nas normas ABNT (2001), sendo semelhante aos modelos anteriormente apresentados para revistas impressas. Como inovação, entretanto, inclui na tabela de avaliação o critério de elementos telemáticos. Nesse item, ela investiga a aplicação de textos em formato HTML e PDF, de ferramentas de busca, de ferramentas interativas (e-mail, formulários), do contador de acessos, de instruções de uso do site, da presença de logotipo de identificação do editor, de políticas de preservação online (armazenamento, recuperação e disseminação) e da multiplicidade de suportes (impresso e CD-ROM).

Fachin (2002) comenta a inexistência prévia na literatura de outro modelo de avaliação que incluísse esses tópicos. Tendo desenvolvido sua pesquisa na área da engenharia, ela os postula com base em estudos de usabilidade e de ergonomia. Anos depois, Mendonça, Fachin e Varvakis (2006) atualizam esse modelo especificamente para avaliação de periódicos online da área da Biblioteconomia. As mudanças no modelo de avaliação tiveram base em Devis (2004), Bomfá (2003), Fachin (2002), Sarmento e Souza (2002) e López-Cózar (1999) e nas normas ABNT (2001; 2003). Em relação à interface amigável e à arquitetura da informação, os autores afirmam que alguns periódicos não mostram preocupação com o uso correto dos mecanismos online e, apesar de estarem parcialmente normalizados, não estão atentos ao conforto visual e informacional dos seus sites - elementos que eles consideram importantes para a atração e para o comprometimento dos usuários.

Fachin, Medeiros e Rados (2008) atualizam novamente a metodologia de Fachin (2002) incluindo agora as normas ISO. Esse novo modelo é aplicado a 17 periódicos científicos online das áreas de Biblioteconomia e de Ciência da Informação. Considerando as inovações da Web 2.0, a análise verifica a presença de ferramentas interativas como chats, fóruns de discussão, opinião do leitor.

Outro trabalho precursor da avaliação de periódicos eletrônicos é de autoria de Sarmento e Souza (2002). Ela apresenta um modelo para análise de estrutura de periódicos científicos eletrônicos a fim de verificar a qualidade destes em comparação à revista impressa. Esse modelo é semelhante ao de Krzyzanowski e Ferreira (1998), mas agrega itens de verificação referentes ao contexto eletrônico, como referências e endereços eletrônicos, data e hora de aceite e inserção do artigo, número de acesso e downloads do artigo, envio do artigo por mala direta, publicação em fluxo contínuo entre outros.

No modelo de Sarmento e Souza (2002) também são apresentados itens de verificação quanto à usabilidade, aos formatos de documentos (texto, imagens e som) e a arquitetura da informação. Para tanto, nele são aplicados critérios postulados por Straioto (2002) a partir de Rosenfeld e Morville (1998). No modelo de Straioto (2002) é investigada a arquitetura da informação de portais acadêmicos e científicos incluindo questões sobre usabilidade, conteúdo e tipos de documento. A validação do modelo ocorreu mediante a avaliação de duas revistas eletrônicas da área da Ciência da Informação.

$\mathrm{Na}$ linha de autores com foco na publicação eletrônica, Oliveira (2005) analisa vinte e sete títulos na área das geociências utilizando os critérios de editoração, de difusão e de visibilidade. No critério de editoração, considera-se a normalização (legenda bibliográfica, ficha catalográfica, ISSN, endereço, normas de publicação, linha editorial) e a gestão editorial (comissão executiva, editor responsável, conselho editorial nacional e internacional). 
No critério difusão, encontram-se os itens publicação (tempo de publicação e regularidade, entidade responsável) e circulação (formas de distribuição, tiragem e recursos financeiros). Por fim, no critério visibilidade, estão itens ligados especialmente ao contexto digital, que são indexação em bases de dados e disponibilização do texto completo na Internet.

No México, López-Ornelas, CorderoArroyo e Backhoff-Escudero (2005) apresentam um sistema digital para avaliar periódicos acadêmicos eletrônicos. A metodologia desenvolvida para o sistema de avaliação é composta de quatro critérios apropriados da tradição impressa: qualidade do conteúdo, padronização, propósito e cobertura, periodicidade e continuidade. Em adição, eles propõem, então, três critérios para avaliação de revistas eletrônicas: a) atualização e manutenção, referente a validade e preservação de links internos e externos da publicação; b) reconhecimento externo do formato digital da publicação, relativo a sua inclusão nas bibliotecas, a sua incorporação nas principais bases de dados da área e a sua obtenção de cobertura e de financiamento; c) navegação e design gráfico: possibilidade do uso de recursos como imagens, cores e suporte gráfico para navegação e estruturação da informação. A Figura 4 apresenta esses critérios e os indicadores correspondentes.

Figura 4: Instrumento de avaliação de periódicos científicos eletrônicos

\begin{tabular}{|c|c|}
\hline Critério & Indicador \\
\hline $\begin{array}{l}\text { Atualização e manutenção: } \\
\text { validade e preservação de links } \\
\text { internos e externos da publicação }\end{array}$ & $\begin{array}{l}\text { - Atualização: } \\
\text { validade dos links internos e externos } \\
\text { da publicação; } \\
\text { - Manutenção: } \\
\text { preservação constante de links internos } \\
\text { e externos da publicação. }\end{array}$ \\
\hline $\begin{array}{l}\text { Reconhecimento externo do } \\
\text { formato digital da publicação: } \\
\text { reconhecimento externo da revista } \\
\text { pela sua inclusão nas bibliotecas; } \\
\text { incorporação nas principais bases } \\
\text { de dados da área; obtenção de } \\
\text { cobertura e financiamento. }\end{array}$ & $\begin{array}{l}\text { - Circulação e formas de distribuição: } \\
\text { vários sistemas de circulação e distribuição } \\
\text { utilizados por uma publicação; } \\
\text { - Inclusão no banco de dados: } \\
\text { a indexação da publicação nas bases de } \\
\text { dados e os índices de visibilidade } \\
\text { nacionais e internacionais em sua área } \\
\text { temática; } \\
\text { - Financiamento: } \\
\text { o reconhecimento que pode ter um } \\
\text { jornal por meio do seu financiamento. }\end{array}$ \\
\hline $\begin{array}{l}\text { Navegaç̧̃ao e design gráfico: } \\
\text { possibilidade do uso de recursos } \\
\text { como imagens, cores e suporte } \\
\text { gráfico para navegação e } \\
\text { estruturaçãa da informação. }\end{array}$ & $\begin{array}{l}\text { - Facilidade de uso: } \\
\text { facilidade de acesso a uma publicação } \\
\text { da Internet; } \\
\text { - Navegação e organização: } \\
\text { organização que facilita a orientação e } \\
\text { o acesso à informação, assim como a } \\
\text { rapidez e a facilidade de circulação em } \\
\text { todo o site; } \\
\text { - Design: } \\
\text { uso de suporte gráfico esteticamente } \\
\text { agradável, em harmonia com o conteúdo; } \\
\text { - Requisitos técnicos: } \\
\text { características do hardware e do } \\
\text { software necessárias para o computador } \\
\text { para acessar os recursos; } \\
\text { - interatividade: } \\
\text { facilidade de interação entre o sistema } \\
\text { e o usuário; } \\
\text { - Conectividade: } \\
\text { tempo de resposta do sistema; } \\
\text { - Capacidade de pesquisa: } \\
\text { presença de sistemas avançados que } \\
\text { facilitalm o acesso e a recuperação } \\
\text { da informação. }\end{array}$ \\
\hline
\end{tabular}

Fonte: Adaptado de López-Ornelas, Cordero-Arroyo e Backhoff-Escudero (2005)

Inf. \& Soc.:Est., João Pessoa, v.28, n.2, p. 209-226, maio/ago. 2018 
A preocupação principal da pesquisa de López-Ornelas, Cordero-Arroyo e BackhoffEscudero (2005) foi a validação do processo de avaliação. Por esse motivo, eles enviaram seu sistema para teste de 16 editores de revistas eletrônicas de diferentes países e de diferentes áreas do conhecimento. Os participantes deveriam julgar o instrumento de pesquisa quanto a clareza, a importância, a relevância e a abrangência de cada indicador e aos critérios em questão. A partir dos resultados recebidos, eles construíram um novo instrumento para ser apresentado ao Conselho Mexicano de Pesquisa Científica a fim de ser aplicado na avaliação das revistas acadêmicas eletrônicas mexicanas.

Gruszynski, Goline e Castedo (2008), a partir da perspectiva da produção editorial, propõem dois roteiros para elaboração de revistas acadêmicas. Um dos roteiros tem foco na produção editorial impressa; o outro, no planejamento de uma revista eletrônica. O objetivo da pesquisa foi verificar os elementos editoriais e gráficos das revistas editadas pelas áreas de Ciências Humanas, de Ciências Sociais Aplicadas, de Linguística, de Letras e de Artes publicadas entre 2003 e 2004 pela Universidade Federal do Rio Grande do Sul. Foram analisados vinte e três títulos. Neles, tanto foi observado o contexto de migração do suporte impresso para o eletrônico, com seus impasses e lacunas, quanto foram propostas novas estratégias de visibilidade do conhecimento.

A diferença desses roteiros para os anteriores, consoante Gruszynski, Golin e Castedo (2008), é que enquanto os demais visaram estabelecer critérios para avaliar os periódicos científicos, esses enfatizaram a produção editorial, ou seja, o planejamento, o estabelecimento de fluxos de edição, bem como a definição de estratégias de circulação. Interessa ao presente estudo a etapa de fluxo de edição dos periódicos online, que apresenta a etapa de edição de leiaute. Nela, estão presentes a definição da arquitetura da informação e da interface do site da revista. Esse item traz como elementos a consistência entre as páginas da revista, os tipos de recursos utilizados, o formato dos fascículos e textos (PDF, html), os sistemas de busca, as ferramentas contextuais (mecanismos de recuperação contextualizada do conteúdo) e a acessibilidade.
Castedo e Gruszynski (2011) aplicam o roteiro de Gruszynski, Golin e Castedo (2008) para análise da produção editorial das revistas brasileiras 'Qualis A' em Comunicação e constatam que, apesar de notarem-se mudanças, a conformação do conhecimento científico ainda está muito atrelada à cultura impressa. Esse estudo toma como base os Elementos da Experiência do Usuário, de Garrett (2003) para desmembrar as etapas de produção das revistas e observa que a tecnologia digital interfere na circulação, mas não no planejamento e no fluxo editorial desses periódicos científicos. A Figura 5 apresenta uma seleção a partir desse roteiro, dos critérios mais relevantes para a presente pesquisa.

Figura 5: Seleção a partir do roteiro de Gruszynski, Golin e Castedo (2008)

\section{Edição de layout}

2.1 Arquitetura da informação e interface do site

Consistência entre as páginas

- Estilos de menus/barras de navegação

- Estilos de texto

- Estilos de cores e imagens

- Estilos de link

Tipos de recursos utilizados pelos artigos

- Texto

- Imagem fixa

- Imagem em movimento

- Áudio

- Outros

Formato dos fascículos e artigos

Sistema de busca

Ferramentas contextuais

Acessibilidade

2.2 Diagramação do fascículo/artigos

2.3 Revisão de provas

2.4 Disponibilização on-line/publicação

Fonte: Adaptado de Gruszynski, Golin e Castedo (2008)

Há também uma outra pesquisa relacionada ao tema arquitetura da informação que traz elementos para a construção de ferramentas para análises de revistas científicas. Francke (2008; 2009) avalia publicações de acesso aberto com interesse de verificar alterações em sua apresentação considerando a transposição do meio impresso para o eletrônico. Ela amplia a avaliação da normalização do artigo considerando itens viáveis no contexto eletrônico, como a possibilidade da URL informada no artigo encaminhar diretamente para o website do autor, por exemplo. Para tanto é aplicada abordagem quantitativa. A 
pesquisa se apoia em conceitos da arquitetura da informação para formatar o que chamou de arquitetura do documento. A pesquisa define arquitetura do documento como "A organização de um documento em termos das estruturas criadas pelos seus elementos constitutivos, por exemplo, em termos de sua relação lógica ou das estruturas que regem o leiaute do documento". (FRANCKE, 2008, p.12).

Francke (2008; 2009) realiza ainda uma avaliação quantitativa da arquitetura da informação como forma de complementar o estudo. Ela faz uma avaliação de quatro revistas diferentes observando aspectos dos sistemas da arquitetura da informação descritos por Rosenfeld e Morville (2002), adaptando-os para a arquitetura do documento. Ela compara a edição mais antiga da revista com a mais recente naquele ano e observa principalmente as diferenças entre $\mathrm{o}$ material impresso e o digital. A autora conclui que as revistas estudadas interpretaram a herança da revista impressa de forma semelhante ao combinála com as convenções de evolução da web.

Nos dias atuais, é possível observar que os modelos clássicos de avaliação ainda têm sido úteis para embasar os estudos sobre a qualidade das revistas, que agora são publicadas preponderantemente em meio digital. Sola e Bonacim (2011) adaptam o modelo de Krzyzanowski e Ferreira (1998) somando a este a metodologia utilizada na avaliação trienal da Capes (2007-2009) com o fim de analisar cinco periódicos nacionais escolhidos de forma aleatória. Os critérios foram Normalização, Duração, Periodicidade, Indexação, Difusão e Colaboração e Divisão do Conteúdo. A avaliação se destinou a classificar as revistas quanto a seu prestígio e qualidade. Assim, atribuiu aos periódicos níveis de qualidade alto, médio e baixo, e - quanto ao âmbito de circulação internacional, nacional, local.

Vishwakarma e Mukherjee (2014) reuniram 30 critérios de avaliação das bases de dados Thomson Reuters, Scopus, SciELO, LISA, LISTA para compor uma ferramenta para as revistas em Biblioteconomia e Ciência da Informação da South Asian Association of Regional Cooperation (SAARC). A pesquisa conclui que, embora haja um número considerável de revistas publicadas na Índia, apenas algumas são qualitativamente fortes. Esse resultado transparece a necessidade constante de avaliação das revistas científicas.
Por fim, tem-se duas pesquisas mais recentes, a pesquisa de Silva (2015) avalia as diferenças entre os formatos de documentos online com base nos princípios funcionais e administrativos do design da informação, nos padrões web, nas recomendações de acessibilidade e usabilidade e nos conceitos de Open Access (acesso aberto). Por sua vez, a pesquisa de Passos (2016) analisa a arquitetura da informação de revistas científicas considerando seus sistemas de busca, organização, navegação, rotulação. Para estudo dos recursos interativos das revistas, a pesquisa postula ainda o sistema de interação.

\section{ANÁLISE DOS RESULTADOS}

Os dados apresentados evidenciam que o tema avaliação de periódicos desperta bastante interesse de pesquisadores brasileiros e latinoamericanos. Muitas vezes financiados pelas agências de fomento, esses autores têm buscado metodologias de avaliação que permitam a classificação dos periódicos, distinguindo aqueles que possuem melhor qualidade e demonstrando onde as verbas disponíveis podem ser melhor empregadas. Os autores da área justificam a motivação para esse interesse pela baixa credibilidade dos periódicos nacionais e pela carência de recursos para financiar a todos os números publicados no país.

Krzyzanowski e Ferreira (1998) e Krzyzanowski, Krieger e Duarte (1991) ressaltavam, na época, a preocupação em âmbito internacional quanto a publicação de periódicos sem qualidade. De forma geral, os veículos eram alvo de críticas devido às irregularidades na publicação e na distribuição; à falta de normalização dos artigos científicos e das revistas como um todo; à falta do corpo editorial e de autoridade da revista. Mais especificamente, em relação às revistas brasileiras, acrescem-se críticas quanto à utilização da língua portuguesa, devido sua baixa penetração no exterior; e quanto ao baixo grau de originalidade e novidade dos artigos científicos publicados.

Devido a esses fatores pejorativos, as revistas brasileiras sofriam com pouca aceitabilidade no meio técnico e científico internacional. Havia também barreiras de acesso à indexação nos índices e bibliografias 
internacionais, os quais balizam sua qualidade por filtros como o Science Citation Index, do ISI (KRZYZANOWSKI; FERREIRA, 1998). A avaliação se fez, pois, importante para destacar os periódicos de qualidade dentre os demais.

Krzyzanowski, Krieger e Duarte (1991) destacavam que os subsídios oferecidos aos editores pelas agências financiadoras no Brasil não eram suficientes para atender a todos interessados. Por essa razão, Krzyzanowski e Ferreira (1998) afirmam a preocupação das agências com a definição de políticas de apoio às revistas científicas, garantindo-se a subsistência das publicações de maior qualidade e a divulgação das pesquisas, que, muitas vezes, são também financiadas por essas agências.

Mais recentemente, Castedo (2009) observa que a definição da prioridade para o recebimento de recursos tem ainda, no início do século XXI, motivado a criação de roteiros de avaliação de periódicos impressos e de eletrônicos. Acrescentam-se a este motivo, outros de ordem financeira que envolvem a avaliação de cursos de pós-graduação, a concessão de bolsas, a progressão funcional, entre outros. Em todos esses fatores, as revistas têm sido utilizadas como indicadores para avaliação, uma vez que reúnem parte da produção dos campos de estudo. Dessa forma, as revistas atuam como índices nos sistemas de julgamento que configuram as estruturas institucionais de pesquisa e, por conseguinte, influenciam nas decisões sobre distribuição de verbas (GRUSZYNSKI; GOLIN; CASTEDO, 2008).

Outro aspecto relevante, em parte também ligado às questões econômicas, é a necessidade dos pesquisadores de publicar em revistas que agreguem prestígio ao seu trabalho. Trzesniak (2006a) lembra que as bancas julgadoras de concurso e de pedidos de auxílios e bolsas, muitas vezes, atribuem valores diferenciados ao currículo dos candidatos dependendo dos veículos nos quais ele publicou sua produção intelectual. Dessa forma, são transmitidos, ao artigo e ao pesquisador, os atributos da revista na qual publicou.

A questão financeira, indubitavelmente, mostra-se como razão definitiva para o amplo interesse no desenvolvimento de instrumentos de avaliação de qualidade em revistas científicas no Brasil. Já em âmbito internacional, os estudos indicam que a principal preocupação é identificar as revistas mais reconhecidas pelos pesquisadores. Assim, por meio de técnicas diversas, os autores consultam seus pares investigando suas percepções a respeito dos periódicos.

A pesquisa mostrou que o intuito principal das pesquisas internacionais é estabelecer uma classificação que aponte quais as principais revistas de cada área. $\mathrm{O}$ interesse se justifica pela importância de indicar para os demais pesquisadores quais revistas poderiam lhes conferir mais prestígio caso conseguissem ter seus artigos nelas publicados. Ademais, o interesse se justifica porque por meio dessas classificações é possível indicar aos autores quais referências poderão ser reconhecidas como de maior credibilidade. Para tal, os autores trabalham com diferentes metodologias, mas, em geral, as pesquisas versam sobre levantamento de reputação dentre os pares, cálculos estatísticos de impacto ou rankings definidos com base em critérios estabelecidos pelas principais bases de dados internacionais.

O Quadro 1 traz uma síntese do que foi apresentado, indicando autores, ano, países de origem, abordagem de pesquisa e temas relacionados. A visualização auxilia na comparação e na análise dos trabalhos. Por meio dessa síntese, confirmam-se as afirmações sobre o interesse de autores brasileiros e latinoamericanos no tema de avaliações de revistas científicas. Destaca-se ainda que a abordagem quantitativa é preponderante nos textos analisados. 
Quadro 1: Pesquisas sobre avaliação de revistas científicas

\begin{tabular}{|c|c|c|c|c|c|c|c|c|c|c|c|c|}
\hline \multicolumn{13}{|c|}{ Pesquisas sobre avaliação de revistas científicas } \\
\hline Ano & Autor & Origem & 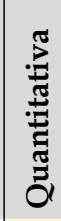 & 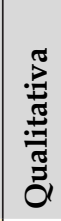 & 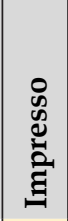 & 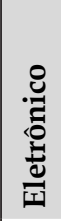 & 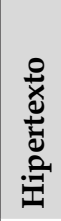 & 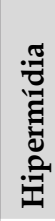 & 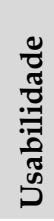 & 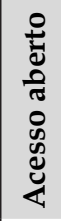 & 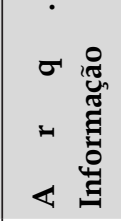 & 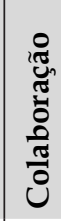 \\
\hline 1982 & Braga e Oberhofer & Brasil & $\mathrm{X}$ & & $\mathrm{x}$ & & & & & & & \\
\hline 1985 & Yahn & Brasil & $\mathrm{x}$ & $\mathrm{X}$ & $x$ & & & & & & & \\
\hline 1986 & Martins & Brasil & $x$ & & $\mathrm{x}$ & & & & & & & \\
\hline 1991 & $\begin{array}{l}\text { Krzyzanowski, Krieger e } \\
\text { Duarte }\end{array}$ & Brasil & $\mathrm{x}$ & & $\mathbf{X}$ & & & & & & & \\
\hline 1994 & Day e Peter & Brasil & & $X$ & $\mathrm{X}$ & & & & & & & \\
\hline 1996 & Castro, Ferreira e Vidili & América Latina & $\mathrm{X}$ & & $\mathbf{X}$ & & & & & & & \\
\hline 1997 & Nkereuwem & Nigéria & & $\mathrm{X}$ & $\mathbf{X}$ & & & & & & & \\
\hline 1997 & López-Cózar & Espanha & $\mathrm{X}$ & & $\mathbf{X}$ & & & & & & & \\
\hline 1998 & Krzyzanowski e Ferreira & Brasil & & $X$ & $\mathbf{x}$ & & & & & & & \\
\hline 1999 & $\begin{array}{l}\text { Yamamoto, Souza e } \\
\text { Yamamoto }\end{array}$ & Brasil & $x$ & & $x$ & & & & & & & \\
\hline 2002 & Yamamoto et al. & Brasil & $\mathrm{X}$ & & & & & & & & & \\
\hline 2002 & Sarmento e Souza & Brasil & $\mathrm{X}$ & & $\mathrm{X}$ & $\mathrm{X}$ & $X$ & $\mathbf{X}$ & $X$ & & $\mathrm{X}$ & \\
\hline 2002 & Fachin & Brasil & $\mathrm{x}$ & & & $x$ & & $x$ & $x$ & & & \\
\hline 2003 & Stumpf & Brasil & $\mathrm{X}$ & & 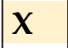 & & & & & & & \\
\hline 2003 & Gorman e Calvert & Brasil & & & & & & & & & & \\
\hline 2003 & Bomfá & Brasil & $\mathrm{X}$ & & $\mathbf{X}$ & & & & & & & \\
\hline 2005 & Oliveira & Brasil & $x$ & & $x$ & $x$ & & & & & & \\
\hline 2005 & $\begin{array}{l}\text { Gruszynski e Sanseverino } \\
\text { López-Ornelas, Cordero- }\end{array}$ & Brasil & & & $x$ & $x$ & & & & & & \\
\hline 2005 & $\begin{array}{l}\text { Arroyo e Backhoff- } \\
\text { Escudero }\end{array}$ & México & $x$ & & & $x$ & $X$ & & $X$ & & $\mathrm{X}$ & \\
\hline $2006 b$ & Trzesniak & Brasil & & & $\mathbf{X}$ & $\mathrm{x}$ & & & & & & \\
\hline 2006 & Bomfá et al. & Brasil & $\mathrm{X}$ & & $\mathbf{X}$ & $x$ & & & & & & \\
\hline 2006 & Fachin e Varvakis & Brasil & $x$ & & & $x$ & & $x$ & $X$ & & & \\
\hline 2007 & Zsidisin et al. & EUA & $\mathrm{X}$ & & $\mathbf{X}$ & & & & & & & \\
\hline 2008 & $\begin{array}{l}\text { Gruszynski, Golin e } \\
\text { Castedo }\end{array}$ & Brasil & $x$ & & $x$ & $x$ & $\mathrm{X}$ & $\mathrm{X}$ & & & $x$ & \\
\hline 2008 & Fachin, Mendonça e Rados & Brasil & $x$ & & & $x$ & & $\mathrm{X}$ & $x$ & & & $x$ \\
\hline 2009 & Francke & Suécia & $x$ & $\mathrm{X}$ & $\mathrm{x}$ & $x$ & $\mathrm{X}$ & & & $\mathrm{X}$ & $\mathrm{x}$ & \\
\hline 2009 & Bolaños-Pizarro et al. & Espanha & $x$ & & $x$ & $\mathrm{x}$ & & & & & & \\
\hline 2009 & Garand et al. & $\begin{array}{l}\text { E U A , C a n a d á, } \\
\text { Inglaterra }\end{array}$ & $x$ & & $x$ & & & & & & & \\
\hline 2010 & Cheng et al. & China & $x$ & & $\mathrm{x}$ & & & & & & & \\
\hline 2010 & Mukherjee & Índia & & $\mathrm{X}$ & & $\mathrm{x}$ & $\mathrm{X}$ & $\mathrm{X}$ & & $\mathrm{X}$ & $x$ & \\
\hline 2011 & Castedo e Gruszynski & Brasil & $x$ & & & $\mathrm{x}$ & $\mathbf{X}$ & $\mathrm{X}$ & $X$ & & $x$ & \\
\hline 2011 & Sola e Bonacim & Brasil & $x$ & & $\mathbf{X}$ & & & & & & & \\
\hline 2012 & Gemser e Friedman & Austrália & $\mathbf{x}$ & & & $\mathbf{X}$ & & & & & & \\
\hline 2014 & Vishwakarma e Mukherjee & Brasil & $\mathrm{x}$ & & & $x$ & & & & & & \\
\hline 2015 & Silva & Brasil & & $\mathrm{X}$ & & $\mathrm{X}$ & & & $\bar{X}$ & $\mathrm{X}$ & & \\
\hline 2016 & Passos & Brasil & & $\mathrm{X}$ & & $\mathrm{X}$ & $\mathbf{X}$ & $\mathrm{X}$ & $\bar{X}$ & & $\mathrm{X}$ & $\mathrm{X}$ \\
\hline
\end{tabular}

Fonte: elaborado pelos autores com base na literatura 
Observou-se que as peculiaridades e os desafios enfrentados pelas revistas científicas dada a sua transposição do meio impresso para o eletrônico foram tema recorrente de trabalhos publicados na última década no século $X X$ e na primeira década do século XXI (GRUSZYNSKI; GOLIN; CASTEDO, 2008; FRANCKE, 2009; CASTEDO; GRUSZYNSKI, 2011). Nos estudos avaliados, verificou-se que a grande maioria dos métodos apresentou critérios semelhantes aos já postulados para avaliação das revistas impressas. Aqueles métodos que consideram o contexto eletrônico, normalmente o fazem adicionando critérios específicos aos tradicionais.

Os temas relacionados às peculiaridades do contexto eletrônico encontrados nas publicações analisadas foram hipertexto, hipermídia, usabilidade, acesso aberto, arquitetura da informação e recursos para interação e colaboração em rede. Sobre o tema interação e recursos para colaboração, foram encontrados apenas dois trabalhos no contexto de avaliações de revistas científicas. Fachin, Mendonça e Rados (2008) incluem em seu instrumento de análise o item ferramentas interativas, que verifica a presença de fórum, de chat e de espaços para opinião do leitor. Esses autores já entendiam como recomendada a presença de tais recursos nas revistas científicas. Contudo, com o avanço da tecnologia e com as novas práticas sociais, ligadas à web, hoje os recursos colaborativos são ainda mais solicitados nos diversos ambientes digitais, como é visto na pesquisa de Passos (2016).

\section{CONSIDERAÇÕES FINAIS}

As pesquisas iniciais a respeito de avaliação de periódicos científicos focalizavam principalmente os aspectos técnicos de qualidade. Desde Braga e Oberhofer (1982) e Krzyzanowski e Ferreira (1998), as principais metas da avaliação concentram-se em verificar a correta normalização das revistas e a adequação a padrões ABNT e ISO. Desde então, as pesquisas sobre avalição de periódicos científicos tem evoluído nos países da américa latina, impulsionadas principalmente por desafios de ordem econômica e pela necessidade de visibilidade em âmbito internacional. Também nos países desenvolvidos, a avaliação das revistas tem se modificado, impulsionada principalmente pelo interesse de hierarquização e ao destaque aqueles que contam com melhor prestígio em suas áreas.

Posteriormente, algumas pesquisas passaram a considerar aspectos de apresentação gráfica (STUMPF, 2003; MENDONÇA; FACHIN, VARVAKIS, 2006; GRUSZYNSKI, GOLIN e CASTEDO, 2008) e mais tarde, motivados pelo novo contexto eletrônico, as avaliações passaram a incluir também elementos de arquitetura de informação e usabilidade (SARMENTO E SOUZA, 2002; FACHIN; MEDEIROS; RADOS, 2008; LÓPEZ-ORNELAS, CORDEROARROYO e BACKHOFF-ESCUDERO, 2005; FRANCKE, 2009; MUKHERJEE, 2010) o que mostra preocupação também com a qualidade funcional dos periódicos, e mais recentemente, há pesquisas preocupadas em agregar recursos interativos às revistas (FACHIN, MENCONÇA, RADOS, 2008; ZUDILOVA-SEINSTRA, 2013; ZUDILOVA-SEINSTRA et al., 2014).

A análise dos resultados permitiu observar que, mesmo que a maior parte das metodologias seja postulada com intuito de avaliar também os periódicos científicos eletrônicos, poucas delas trazem critérios que contemplam em amplitude as especificidades do meio digital. Verifica-se então a necessidade de estudos que incluam as questões de qualidade referentes às características específicas das revistas científicas, adquiridas mediante sua transposição para $o$ meio eletrônico.

A pesquisa mostra também a busca incessante da comunidade científica pela qualificação dos seus principais meios de comunicação, que são as revistas. $O$ interesse crescente dos autores nos temas hipertexto, hipermídia, usabilidade, acesso aberto, arquitetura da informação e recursos para interação e colaboração em rede relacionados às revistas indica que tais temas são considerados relevantes pelos pesquisadores. Ao mesmo tempo, os resultados mostram alguns assuntos que ainda não foram explorados em profundidade.

Concluindo, a pesquisa traça uma tendência de renovação das metodologias de avaliação com vistas à sua adequação às novas demandas da publicação digital. Mostra-se evidente a preocupação dos pesquisadores com a necessidade de constante atualização dos métodos de avaliação, acompanhando o avanço 
da tecnologia e a sua apropriação por parte da comunidade científica. Nesse sentido, entendese que este estudo seja útil para orientar novas pesquisas quanto aos principais temas que ainda devem ser abordados visando o aprimoramento dos periódicos científicos.

Artigo recebido em 08/04/2016 e aceito para publicação em 03/05/20।8

\section{QUALITY CRITERIA IN SCIENTIFIC JOURNALS: a study on the adequacy to the digital environment}

ABSTRACT The evaluation of scientific journals has been a concern of researchers since the mid-twentieth century. The evaluation criteria initially proposed considered the quality of scientific journals in their original support, the form. However, over time, these magazines have adopted mainly the digital medium for publication. In this context, it is interesting to the present research to investigate to what extent the evaluation of the scientific journals has accompanied the technological advance, also covering aspects related to the electronic support. The question that guides the research is as follows: how do the evaluation methodologies for scientific journals contemplate digital media criteria? To answer this question, we identify, in publications relating to the theme, quality criteria of scientific journals associated with the specifics of the electronic media. Data collection for this purpose was done at the Web of Science, Science Direct and Brapci databases, at the Brazilian Digital Library of Theses and Dissertations, at the Thesis Bank and at the Google Scholar. As a resource for the presentation of results, a comparative table of evaluation methodologies is presented, highlighting a presence of the criteria: print media, electronic media, hypertext, hypermedia, usability, open access, information architecture and resources for network interaction and collaboration. The table also informs about the authors that gave origin to related studies and classifies the methodologies as qualitative or quantitative. The research demonstrates a tendency to adapt the methodologies of evaluation of scientific journals to the digital context and presents areas that are still available.

Keywords: $\quad$ Scientific journal. Quality assessment. Information Architecture.

\section{REFERÊNCIAS}

ASSOCIAÇÃO BRASILEIRA DE NORMAS TÉCNICAS (ABNT). NBR 6022: Informação e Documentação - Artigo em publicação periódica científica impressa - Apresentação. Rio de Janeiro, 2003. 5 p.

ASSOCIAÇÃO BRASILEIRA DE NORMAS TÉCNICAS (ABNT). Projeto NBR 6021: apresentação de publicações periódicas. Rio de Janeiro: ABNT, 2001.

BOLAÑOS-PIZARRO, M. et al. Evaluación formal de las revistas españolas del área del sistema cardiovascular. Clin Invest Arterioscl, v. 21, n. 1, p. 34-41, 2009.
BRAGA, G. M.; OBERHOFER, A. Diretrizes para avaliação de periódicos científicos e técnicos brasileiros. Revista Latinoamericana de Documentación, , v. 2, n. 1, p. 27-31, 1982.

BOMFÁ, C. R. Z. Publicação de revistas científicas em mídia digital: critérios e procedimentos. Florianópolis: Visual Books, 2003.

COORDENAÇÂO DE APERFEIÇOAMENTO
DE PESSOAL DE NÍVEL SUPERIOR
(CAPES). Qualis Periódicos. 2015.
Disponível em: <https://sucupira.capes.
gov.br/sucupira/public/consultas /
coleta/veiculo Publicaca o Q a li s /
listaConsultaGeralPeriodicos.jsf >. Acesso em 5
out. 2015.

CASTEDO, R. S. Revistas científicas on-line de comunicação no Brasil: a produção editorial 
sob o impacto da tecnologia digital. 2009. 120f. : il. Dissertação (mestrado) -Universidade Federal do Rio Grande do Sul, Faculdade de Biblioteconomia e Comunicação, Programa de Pós-Graduação em Comunicação e Informação. Porto Alegre, RS, 2009. Disponível em: <http:// www.raquelcastedo.com/textos/dissertacao. html>. Acesso em: 5 out. 2014.

CASTEDO, R.S.; GRUSZYNSKI, A. C. A produção editorial de revistas científicas on-line: uma análise de publicações brasileiras da área da Comunicação. Revista Em Questão, Porto Alegre, v. 17, n. 1, p. 271-287, 2011. Disponível em: $\quad<$ http://seer.ufrgs.br/index.php/ EmQuestao/article/view/16697/12482>. Acesso em: 5 out. 2014.

CASTRO, R. C. F.; FERREIRA, M. C. G.; VIDILI, A.L. Periódicos latino-americanos: avaliação das características formais e sua relação com a qualidade científica. Ciência da Informação, Brasília, v. 25, n. 31996.

CHENG, S. et al. PrestigeRank: A new evaluation method for papers and journals, 2010. Journal of Informetrics, v. 5, n. 1, p. 1-13, 2011.

CHRESSANTHIS, G. A.; CHRESSANTHIS, J. D. The relationship between manuscript submission fees and journal quality. Serials Librarian. V. 24, n.1, p. 71-86, 1993.

DAY, A.; PETERS, J. Quality Indicators in Academic Publishing. Library Review, v. 43, n. 7 p. 4-72, 1994.

DEVIS, J. D. et al. Las revistas científico-técnicas españolas de ciencias de la actividad física y el deporte: adecuación a las normas ISO y grado de normalización. Ciência da Informação, Brasília, v. 33, n. 1, p. 38-47, jan./abr. 2004. Disponível em: <https:/ /www.researchgate.net/ publication/45363754_Las_revistas_cientificastecnicas_espanolas_de_las_ciencias_de_la_ Actividad_Fisica_y_el_Deporte_inventario_y_ analisis_de_la_calidad_de_contenido_y_difusion >. Acesso em: 5 out. 2015.

FACHIN, G. R. B. Modelo de avaliação para periódicos científicos on-line: proposta de indicadores bibliográficos e telemáticos 2002. 210 f. Dissertação (Mestrado em Engenharia de Produção) - Programa de Pós-Graduação em Engenharia de Produção, Universidade Federal de Sant Catarina, Florianópolis, 2002.

FACHIN, G. R. B.; MEDEIROS, G. M.; RADOS, G. J. V. Padronização de periódicos científicos on-line da área de Biblioteconomia e Ciência da Informação: adequação às normas ISO. Revista ACB: Biblioteconomia em Santa Catarina, Florianópolis, v. 13, n. 2, p. 415-438, jul/dez. 2008. Disponível em: <http://www. acbsc.org.br/revista/index.php/racb/article/ viewFile/539/667>. Acesso em: 5 out. 2015.

FERREIRA, M. C. G. Avaliação de periódicos científicos. In: FÓRUM NACIONAL DE PADRONIZAÇÃO E DIVULGAÇÃO DA PRODUÇÃO CIENTÍFICA, 2001. Conferência. Fortaleza, 2001. Disponível em: <http://www. biblioteca.ufc.br/forum.html>. Acesso em: 09 set. 2015.

FRANCKE, H. (Re)creations of Scholarly Journals Document and Information Architecture in Open Access Journals distribution. Valfrid, 2008. Disponível em: <http://hdl.handle.net/2320/1815>. Acesso em: 10 set. 2015 ..

Towards an architectural document analysis. Journal of Information Architecture, v. 1, n. 1, 2009.

GARAND, J. C.; GILES, M. W.; BLAIS, A.; MCLEAN, I.. Political science journals in comparative perspective: evaluating scholarly journals in the United States, Canada, and the United Kingdom, 2009. Political Science and Politics, v. 42, n. 4, p. 695-717, oct 2009.

GARRETT, J. J. The elements of user experience: user centered design for the Web. New York/ Berkeley: Aiga/New Riders, 2003.

GEMSER, G.; FRIEDMAN, K.; Quality perceptions of design journals: the design scholars' perspective. Design Studies, v. 33, n. 1, jan. 2012. 
GORMAN, G. E. Library and information science journals in the Asian context. In: IFLA COUNCIL AND GENERAL CONFERENCE, 65. The Hague, 1999: Papers and proceedings. The Hague, 1999.

GORMAN, G. E., CALVERT, P.J. LIS journal quality: results of a study for the IFLA library and information science journals. In: IFLA General Conference and Council, 69., Berlin, 2003. Proceedings. Berlin, 2003.

GREENE, L. J. Mais visibilidade para a ciência brasileira: biblioteca eletrônica já reúne mais de 50 revistas científicas. Revista Fapesp, n57, set. 2000. Disponível em: <http://revistapesquisa. fapesp.br/2000/09/01/mais-visibilidade-para-aciencia-brasileira/>. Acesso em: 5 jul. 2015.

GRUSZYNSKI, A. C.; GOLIN, C.; CASTEDO, R. Produção editorial e comunicação científica: uma proposta para edição de revistas científicas. E-Compós, Brasília, v.11, n.2, 2008. Disponível em: < http://www.compos.org.br/seer/index. php/e-compos/article/view/238>. Acesso em: 22 jul. 2013.

GUÉDON, J.-C. Digitalizar las revistas científicas. ¿Cómo garantizar el acceso universal a la información científica básica? Mundo Científico, n. 218, p. 80-87, dec. 2000.

KRZYZANOWSKI, R. F.; FERREIRA, M. C. G. Avaliação de periódicos científicos e técnicos brasileiros. Ciência da Informação, Brasília, v.27, n. 2, p.165-175, maio/ago., 1998.

KRZYZANOWSKI, R. F.; KRIEGER, E. M.; DUARTE, F. A. M. Programa de apoio às revistas científicas para a Fapesp. Ciência da Informação, Brasília, v. 20, n. 2, p. 137-150, jul./dez. 1991.

LÓPEZ-CÓZAR, E. D. Iso Standars for the presentation of scientific periodicals: little known and little used by Spanish biomedical journals. Journal of Documentation , [S. 1.], v. 55, n.3, jun. 1999.

Evaluación del grado de de ajuste de las revistas españolas de ciencias de la salud a las normas internacionales de presentación de publicaciones periódicas. Revista Española de Salud Publica, v. 71, n. 6, 1997.

LÓPEZ-ORNELAS, M.; CORDERO-ARROYO, G.; BACKHOFF-ESCUDERO, E. Measuring the Quality of Electronic Journals. The Electronic Journal of Information Systems Evaluation, v. 8, n. 2, p. 133-142, 2005.

MARTINS, M. D. Avaliação da normalização de periódicos brasileiros nas áreas de ciência e tecnologia. Revista de Biblioteconomia de Brasília, v.14, p.197-208, 1986.

MENDONÇA, T. C.; FACHIN, G. R. B.; VARVAKIS, G. Padronização de periódicos científicos on-line: estudo aplicado na área de Biblioteconomia e Ciência da Informação. Informação \& Sociedade: Estudos, João Pessoa, v. 16, p. 1-27, 2006.

MUKHERJEE, B. Scholarly communication in library and information services.: the impacts of Open Access journals and e-journals on a changing scenario. Oxford: Elsevier Science,. Chandos Publishing, 2010.

NKEREUWEM, E. E. Accrediting knowledge, Library Review, v. 46, n. 2, p. 99-104, 1997.

OLIVEIRA, É. Produção científica nacional na área de geociências: análise de critérios de editoração, difusão e indexação em bases de dados. Ciência da Informação, Brasília, v. 34, n. 2, p. 34-42, maio/ago. 2005.

PACKER, A. L. et al. SciELO: uma metodologia para publicação eletrônica. Ciência da Informação, Brasília, v. 27, n. 2, p. 109-212, 1998. Disponível em: <http://www.scielo. br $/$ scielo.php?script $=$ sci_arttext\&pid $=$ S0100$19651998000200002 \& \operatorname{lng}=\mathrm{pt} \& \mathrm{nrm}=$ iso\&tlng $=\mathrm{pt}>$. Acesso em: 5 jul. 2015.

REVISTA PESQUISA FAPESP. SciELO, um modelo reconhecido: biblioteca virtual amplia visibilidade da ciência brasileira. Revista Pesquisa FAPESP, n. 73, mar. 2002. Disponível em: <http://revistapesquisa.fapesp. 
br/2000/09/01/mais-visibilidade-para-a-cienciabrasileira>. Acesso em: 5 jul. 2015.

ROSENFELD, L.; MORVILLE, P. Information architecture for the World Wide Web. Sebastopol: O’Really, 1998.

ROSENFELD, L.; MORVILLE, P.. Information architecture for the World Wide Web. 2. ed. Sebastopol: O'Really, 2002.

SARMENTO E SOUZA, M. F. Periódicos científicos eletrônicos: apresentação de modelo para análise de estrutura. 2002. 154f. Dissertação (Mestrado em Ciência da Informação) Faculdade de Filosofia e Ciências, Universidade Estadual Paulista. Marília. 2002.

SCIENTIFIC ELETRONIC LIBRARY (SCIELO). Sobre o SciELO. 2015 . Disponível em: <http:// www.scielo.org>. Acesso em: 5 jul. 2015.

SOLA, G. L.; BONACIM, C. A. G. Avaliação bibliométrica de periódicos brasileiros: contrastando a metodologia Qualis-CAPES com o Modelo de Krzyzanowski e Ferreira (1998). In: ENCONTRO DA ANPAD, 35., 2011, Rio de Janeiro. Anais... Rio de Janeiro, 2011.

SOUZA, E. P.; PAULA, M. C. S. Qualis: a base de qualificação dos periódicos científicos utilizada na avaliação CAPES. InfoCAPES Boletim Informativo, v. 10, n. 2, abr./jun. 2002.

STRAIOTO, F. A arquitetura da informação para a Word Wide Web: um estudo exploratório. 2002. 125f. Dissertação (Mestrado em Ciência da Informação) - Faculdade de Filosofia e Ciências, Universidade Estadual Paulista, Marília, 2002.

STUMPF, I. R. C. Avaliação das revistas de comunicação pela comunidade acadêmica da área. Em Questão: revista da Faculdade de Biblioteconomia e Comunicação da UFRGS, Porto Alegre, v. 9, n. 1, p. 25-38, jan./jun. 2003.

TESTA, J. The ISI database: The journal selection process.1998. . Disponível em: <<http://cs.nju. edu.cn/gchen/isi/help/HowToSelectJournals. html>. Acesso em: 16 set. 2014.
TRZESNIAK, P. As dimensões da qualidade dos periódicos científicos e sua presença em um instrumento da área da educação. Revista Brasileira de Educação, v. 11, n. 32, maio/ago. 2006a.

TRZESNIAK, P. A avaliação de revistas eletrônicas para órgãos de fomento: respondendo ao desafio. In: CONFERÊNCIA IBEROAMERICANA DE PUBLICAÇÕES ELETRÔNICAS NO CONTEXTO DA COMUNICACÃO CIENTÍFICA, 1., 2006, Brasília. Anais... Brasília: Universidade de Brasília, 2006b.

VISHWAKARMA, P.; MUKHERJEE, B. Developing Qualitative Indicators for Journal Evaluation: Case Study of Library Science Journals of SAARC Countries. Journal of Library \& Information Technology, v. 34, n. 2, mar. 2014.

YAHN, V. G. Avaliação de periódicos brasileiros de agricultura. Rev Bras Bibliotecon Doc, v. 18, p.39-53, 1985.

YAMAMOTO, O. et al. Avaliação de periódicos científicos brasileiros da área da Psicologia. Ciência da Informação, Brasília, v. 31, n. 2, p. 163-177, maio/ago. 2002.

YAMOMOTO, O. H.; SOUZA, C. C.; YAMAMOTO, M. E. A produção científica na psicologia: uma análise dos periódicos brasileiros no período 1990-1997. Psicologia Reflexão e Crítica, v. 12, n. 2, p. 549-565, 1999.

YU, L. et al. Scholarly journal evaluation based on panel data analysis. Journal of Informetrics, v. 3, p. 312-320, 2009.

ZSIDISIN, G.A. et al. Evaluation criteria development and assessment of purchasing and supply management journals. Journal of Operations Management, 25 (2007) 165-183.

ZUDILOVA-SEINSTRA, E. et al. The Elsevier Article of the Future project: a novel experience of online Reading. In: COPE, B.; PHILLIPS, A.(Edt.). The Future of the academic journal. 2nd. ed.). Chandos Publishing, 2014. p. 357-377. 